PDFlib PLOP: PDF Linearization, Optimization, Protection

Page inserted by evaluation version www.pdflib.com - sales@pdflib.com 


\title{
Characteristics of Cocaine Users Presenting to an Emergency Department Chest Pain Observation Unit
}

\author{
Brenda M. Booth, PhD, Jim Edward Weber, DO, Maureen A. Walton, MPH, PhD, \\ Rebecca M. Cunningham, MD, Lynn Massey, MSW, CSW, Carol R. Thrush, MA, \\ Ronald F. Maio, DO, MS
}

\begin{abstract}
Objectives: This report examines the sociodemographic and substance use characteristics, co-occurring psychological status, substance abuse consequences, and prior experiences with substance abuse treatment among patients with cocaine-associated chest pain presenting to an emergency department chest pain observation unit. Methods: This was a consecutive cohort of patients in the emergency department chest pain observation unit aged 18-60 years with low to moderate risk for acute coronary syndrome and recent cocaine use. Responses on standardized and validated instruments were used to examine demographic and clinical characteristics of the sample and to compare patients who met Diagnostic and Statistical Manual of Mental Disorders, Fourth Edition (DSM-IV) criteria for past three-month substance abuse or substance dependence with patients who did not. Results: Of 145 eligible patients identified between June 1, 2002, and February 29, 2004, 86\% met criteria for a lifetime DSM-IV substance use disorder

and 50\% met past three-month criteria. Approximately one half of the total sample reported substantial symptoms of depression. Substance use frequency and consequences, depression, and psychological distress were significantly more severe among those with past three-month substance use diagnoses; however, most sociodemographic characteristics were not associated with substance use diagnoses. Interest in treatment services and treatment history was also significantly associated with the presence of a substance use disorder diagnosis. Conclusions: Findings regarding diversity in alcohol and drug involvement, current level of psychological functioning, depressive symptomatology, and interest in treatment services provide useful information for designing emergency department-based interventions for this population. Key words: cocaine; chest pain; emergency department; demography; substance-related disorders; behavioral symptoms. ACADEMIC EMERGENCY MEDICINE 2005; 12:329-337.
\end{abstract}

Cocaine is the most common illicit drug of abuse in patients who present to the emergency department (ED), and cocaine-related ED cases have increased $47 \%$ from 1995 to $2002 .{ }^{1}$ Correspondingly, as cocaine use has become more widespread, the number of cocaine-related cardiovascular events has increased. Chest pain, whether cardiac or noncardiac, is the most frequently reported consequence of cocaine abuse in the $\mathrm{ED}^{2}$ and is highly prevalent in urban, inner-city EDs. ${ }^{3,4}$ Associated with this increased use of cocaine

From the Division of Health Services Research, Department of Psychiatry and Behavioral Sciences, College of Medicine, University of Arkansas for Medical Sciences (BMB, CRT), Little Rock, AR; Central Arkansas Veterans Healthcare System (BMB, CRT), Little Rock, AR; Departments of Emergency Medicine (JEW, RMC, RFM) and Psychiatry (MAW, LM), University of Michigan, Ann Arbor, MI; and Hurley Medical Center (JEW), Flint, MI.

Received June 17, 2004; revision received October 29, 2004; accepted November 4, 2004.

Supported by the National Institute on Drug Abuse (RO1 grant DA14343).

Address for correspondence and reprints: Brenda $\mathrm{M}$. Booth, $\mathrm{PhD}$, Division of Health Services Research, Department of Psychiatry and Behavioral Sciences, University of Arkansas for Medical Sciences, 5800 West 10th Street, Suite 605, Little Rock, AR 72204. Fax: 501-660-7542; e-mail: boothbrendam@uams.edu.

doi:10.1197/j.aem.2004.11.021 is the evaluation of more than 64,000 cocaine-using patients annually for possible myocardial ischemia at an annual cost in 1995 dollars of more than $\$ 83$ million. ${ }^{5}$

The cardiovascular consequences of cocaine use are cogently described. The risk of acute myocardial infarction (AMI) is increased 24-fold in the hour after cocaine use, ${ }^{6}$ and up to $6 \%$ of patients who present to the ED with cocaine-induced chest pain sustain an AMI. $^{4,5}$ In addition, cocaine accounts for up to $25 \%$ of AMIs in patients 18-45 years of age, and users have a lifetime risk of nonfatal AMI that is seven times the risk of nonusers. ${ }^{7}$ Numerous factors have been implicated in the pathophysiology of cocaine-associated myocardial ischemia, including coronary artery vasoconstriction, ${ }^{8}$ platelet aggregation, ${ }^{9-12}$ in situ thrombus formation, ${ }^{13-16}$ and premature atherosclerosis. ${ }^{10,15,17,18}$ However, the relative contributions of each of these mechanisms have not been widely investigated and are poorly understood.

Even less is known about the clinical picture of cocaine users presenting to the ED with chest pain in terms of their overall substance use, their severity of substance use, and their interest in treatment. Previous studies have focused on individuals with substance abuse and concomitant trauma and injuries. ${ }^{19,20}$ In 
addition, previous research has established that psychiatric disorders are common comorbid conditions among cocaine abusers in substance abuse treatment. ${ }^{21}$ However, there is a paucity of literature regarding psychiatric disorders and other sociodemographic and psychosocial characteristics among cocaine users who are not in treatment who present to the ED with chest pain.

The majority of patients with cocaine-associated chest pain who are at low to moderate risk for cardiac complications can be treated in ED chest pain observation units (CPOUs), and the safety and feasibility of treatment protocols for these patients in CPOUs have been previously demonstrated. ${ }^{22}$ We present a unique and comprehensive characterization of the substance use, mental health, and treatment utilization characteristics of cocaine-using patients who presented to an ED with a chief complaint of chest pain and who were admitted to the CPOU for observation and treatment. The ED serves as a "window of opportunity" for identification and early intervention for substance abusers. ${ }^{23}$ Therefore, this information regarding the characteristics of cocaine users who present to the ED with chest pain may aid in the development of EDbased intervention protocols. In addition to providing basic descriptive information about the characteristics of these patients, this report also contrasts subgroups of patients based on the presence of a substance use diagnosis in the three months just before ED admission.

\section{METHODS}

Study Design. This study used a consecutive cohort design. It was approved by the investigators' institutional review boards.

Study Setting and Population. This study was conducted at an urban, university-affiliated Level I trauma center with an annual ED census of approximately 75,000 patients per year. Patients presenting to an ED CPOU with chest pain and recent cocaine use were included.

CPOU Protocol. The institutional standard of care where the study was conducted requires that patients younger than 60 years of age who receive a workup for potential acute coronary syndrome (ACS) also undergo urine toxicologic screening for cocaine metabolites (Synchron LX Systems, Fullerton, CA). A detailed description of the CPOU protocol has previously been reported. ${ }^{24}$ Briefly, a cardiac troponin I level (Access Accu TnI; Beckman Coulter, Fullerton, CA) was measured at presentation and at three, six, and nine hours. The threshold level of $0.5 \mathrm{ng} / \mathrm{dL}$ was the institutional cutoff criteria. Cocaine use was confirmed with toxicologic-positive urine screening for cocaine metabolites (Synchron LX Systems). The relative sensitivity $(95 \%)$ and specificity $(100 \%)$ of the urine cocaine metabolite assay are excellent (Synchron LX Systems). ${ }^{25}$

Sample Recruitment. To maximize patient recruitment, members of the research staff were present in the ED CPOU between 8 AM and 10 PM seven days per week. The rationale for not deploying staff to recruit patients during the midnight shift was based on results from our pilot work indicating that patients on this shift were generally sleeping and could not be awakened to complete baseline assessments. Further, because the institutional standard adopted by this ED includes provisions for a nine-hour monitoring period to assess low- to intermediate-risk patients with chest pain (e.g., not at high risk for ACS) in the CPOU, eligible patients often could be recruited by staff the following morning. We used a two-phase enrollment procedure.

Phase 1: Initial Screening. All consecutive patients aged $18-60$ years in the CPOU were initially considered eligible for the study and approached by research staff to participate in the screening during the recruitment period (June 1, 2002, to February 29, 2004). Research staff reviewed ED logs to identify CPOU patients to participate in the screening. Research staff maintained daily logs documenting the classification of potential patients as either approached for the study and subsequently screened, refused, or missed (e.g., not approached before leaving the ED). Each week, the field supervisor conducted a quality review of the logs to verify that every patient entering the $\mathrm{ED}$ with chest pain was accounted for in the data.

For the initial screening, research staff obtained written informed consent to view patient medical records for determination of study eligibility. As an incentive for the screening phase, patients who provided written informed consent to allow research staff to review their medical records could choose a $\$ 1$ item from a gift basket containing items from a local "dollar" store (e.g., lotion, tape measure, flashlight, storage containers). A research social worker reviewed consenting participants' charts and documented the inclusion/exclusion criteria to determine eligibility for the main study.

Inclusion Criteria. Inclusion criteria included age 18-60 years and a positive toxicologic urine screen for cocaine or, if urine screen results were incomplete or unavailable, physician documentation in the medical chart indicating that the patient was treated for cocaine-related chest pain and patient self-reported cocaine use.

Exclusion Criteria. Because the study was focused on the CPOU, high-risk patients were ineligible and were defined as those admitted to the critical care unit or telemetry unit; patients with an initial electrocardio- 
gram suggestive of ischemia, AMI, or ST-segment elevation or depression of $1 \mathrm{~mm}$ or more that persisted for at least 1 minute; elevated serum levels of cardiac markers; recurrent ischemic chest pain; a history of AMI or coronary artery bypass surgery; hemodynamic instability; receiving anticoagulant therapy in the ED (e.g., heparin, enoxaparin); or receiving intravenous vasoactive medications (nitroglycerine, nitroprusside). Patients were also ineligible for the study if they did not undergo a cardiac workup for an ACS (e.g., local trauma, radiographic abnormalities, or other clearly noncardiac causes) or if they were pregnant, unable to provide informed consent (e.g., unconscious, incarcerated, or did not comprehend the English language), or acutely suicidal (resulting in constant presence of designated hospital staff, agitation, or violence while in the ED requiring restraint or hospital security monitoring).

Phase 2: Main Study Enrollment. Subjects who participated in the screening phase, met inclusion criteria, and did not meet any exclusion criteria were approached to participate in phase 2. Patients with negative urine drug screens for cocaine were not approached to participate in the study. After signing a written consent form, a two-hour baseline interview was conducted by trained research staff during the patient's CPOU stay and included questions about demographics, substance use history, and mental health (see Measures). After completing the baseline assessment, all study participants were given referral information for local substance abuse treatment services and human immunodeficiency virus testing. Remuneration for the baseline assessment was a $\$ 25 \mathrm{gift}$ certificate to a local retailer (e.g., Wal-Mart, Target).

Measures. The baseline assessment contained a variety of measures, including the Substance Abuse Outcomes Module (SAOM), ${ }^{26,27}$ the Brief Symptom Inventory (BSI) to assess general psychological distress, ${ }^{28}$ and the Patient Health Questionnaire (PHQ-9) to assess depression symptomatology. ${ }^{29,30}$

The SAOM is designed for evaluation of substance abuse treatment outcomes (http://www.netoutcomes. net) and measures Diagnostic and Statistical Manual of Mental Disorders, Fourth Edition (DSM-IV) substance use disorders, ${ }^{31}$ a variety of key prognostic factors for treatment outcome, and outcome domains. For the current study, the SAOM was used to measure basic demographic information, including age, gender, race, marital status, education, and employment status as well as information on lifetime and recent (past three months) substance use diagnoses, substance abuse treatment history, antisocial personality disorder, and substance-related consequences. ${ }^{32}$

The SAOM takes approximately 30 minutes to complete and was administered by trained research assistants. The SAOM has undergone extensive re- liability and validity examinations and demonstrates reasonable reliability (internal reliability, coefficient $\alpha$, $0.58-0.90$; test-retest reliability, $0.56-0.99$ ) and validity (concurrent validity generally $0.5-0.8$; predictive validity, 0.5-0.9). ${ }^{33}$ Concurrent validity for the SAOM was based on longer key instruments such as a structured diagnostic interview for substance use disorders, the Composite International Diagnostic Interview-Substance Abuse Module (CIDI-SAM), ${ }^{34}$ and the Addiction Severity Index. ${ }^{35}$ The SAOM has shown $90 \%-93 \%$ agreement with the CIDI-SAM on DSM-IV substance use diagnosis (present/absent). ${ }^{33}$

The SAOM measures substance-related consequences using a 15-item index derived from the Inventory of Drug Use Consequences (InDUC-2R) instrument designed to assess lifetime and recent drug and/or alcohol use consequences. ${ }^{32}$ Specifically, five subscales (three items each) assess physical, intrapersonal, interpersonal, impulse control, and social consequences of substance use.

Self-reported use of substance abuse treatment services included lifetime and past-year use of formal specialty treatment services and/or informal services (e.g., self-help groups) as well as past-year report of any mental health-related treatment or treatment for depression.

The $\mathrm{BSI}^{28}$ was used to measure psychological distress and includes three subscales that are labeled depression, anxiety, and the global severity index. The BSI has demonstrated good test-retest reliability for the subscales (reliabilities of 0.68-0.91), high internal consistency ratings (coefficient $\alpha$ of $0.71-0.85$ ), and sensitivity to change. ${ }^{28}$ In addition, the PHQ-929,30 was used as a more detailed examination of depression severity and symptomatology. The PHQ-9 scores each of the nine DSM-IV criteria for depression as zero (not at all) to three (nearly every day). Substantial symptoms of depression are indicated by PHQ-9 scores $>12$. The PHQ-9 has $94 \%$ specificity and $99 \%$ sensitivity for current major depressive disorder in a primary care population. ${ }^{29,30}$ PHQ-9 total scores range from zero to 27 with severity defined as follows: none (0-5), mild (6-10), moderate (11-15), major (16-20), severe $(21+)$.

Staff Training. Training for research staff was provided, including mock sessions in administration of the interview instruments with the field coordinator and observation of interviews with patients until acceptable competence with the interview was achieved. Periodic retraining sessions to maintain the integrity of the study protocol were also provided to research staff, and reliability checks on interviewers included regular review for out-of-range values and error reports that were generated and reviewed with staff.

Data Analysis. Descriptive statistics were computed for basic sociodemographic, service use, substance 
use, and psychological characteristics. Bivariate analyses were computed to compare participants who met criteria for either a DSM-IV substance abuse or dependence diagnosis in the past three months with those who did not meet these criteria; specifically, analysis of variance was used for continuous measures and chi-square tests for categorical measures. We chose to classify patients by diagnoses of substance use disorders in general, rather than by cocaine use disorders specifically, in order to investigate their entire addiction severity, which included alcohol and marijuana abuse and dependence for many patients. In fact, results were very similar when classifying patients by substance use or cocaine disorders except that differences were stronger for the former because the "no cocaine diagnosis" group included individuals with alcohol or other substance use disorders. SAS software (version 8.2; SAS Institute, Inc., Cary, NC) was used for statistical analyses.

\section{RESULTS}

Figure 1 provides a summary of the study recruitment efforts for phase 1 and phase 2. Of 1,437 total patients with chest pain eligible for phase 1 (which involved consent for research staff to view their medical record), $94 \%(n=1,343)$ agreed to participate, $4 \%$ $(n=63)$ refused, and 2\% $(n=31)$ were missed (e.g., admitted and discharged during the shift not covered or staff simply missed subject). The primary reasons noted by research staff for patient refusal to participate in phase 1 were due to patient concerns about confidentiality or invasion of privacy $(n=22)$, the patient simply did not want to participate $(n=14)$, and reasons related to severe pain, fatigue, or emotional stress $(n=16)$ or other reasons $(n=11)$. Our local institutional review board did not permit review of medical records for patients who did not consent to participate in phase 1 of the study. Therefore, it was not possible to determine whether subjects refusing to participate in phase 1 of the consent process differed from consenting patients.

Of the 1,343 who agreed to participate in phase 1 , urine cocaine screens were completed on 1,192 (89\%), of which $205(18 \%)$ were positive and thus eligible to participate in phase 2 of the study. Fifty-two cocainepositive patients were deemed ineligible due to receiving anticoagulant therapy or intravenous vaso-

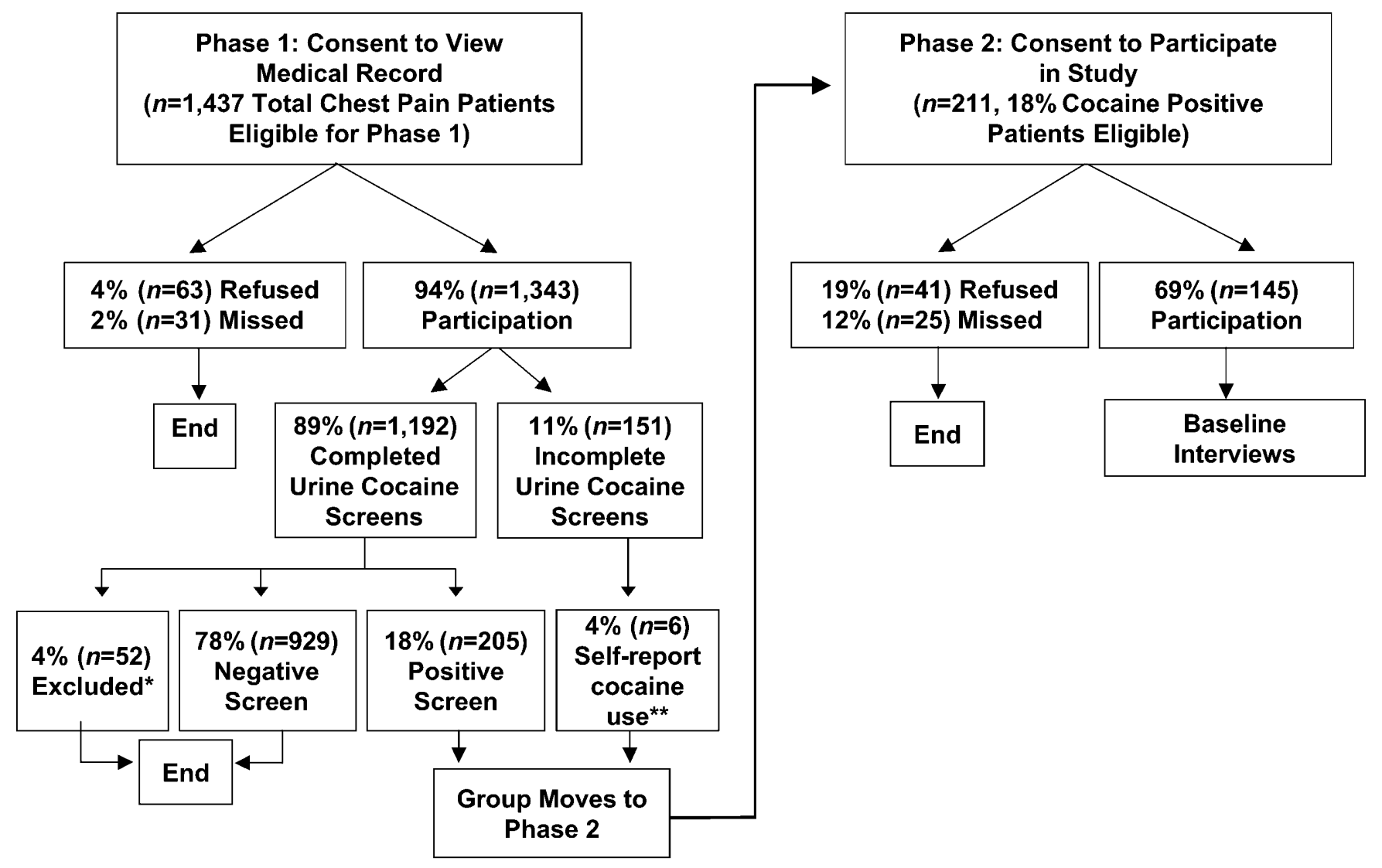

*Positive toxicology screen for cocaine, but patients met high risk criteria.

**Patient self-reported cocaine use and physician documentation in chart indicated patient being treated for cocaine-related chest pain.

Figure 1. Recruitment of participants in the chest pain observation unit for the cocaine chest pain study between June 1, 2002, and February 29, 2004. 
active medications $(n=27)$, evidence indicating previous history of myocardial infarction or coronary artery bypass grafting $(n=10)$, or other reasons $(n=15)$. Urine cocaine screens were incomplete for an additional six patients who were also considered eligible for phase 2 because they had reported recent cocaine use that was corroborated by physician documentation in their medical record indicating they were being treated for cocaine-related chest pain. Of these 211 individuals with recent cocaine use, $69 \%$ agreed to participate $(n=145), 19 \%$ refused, and $12 \%$ were missed by recruitment staff for various reasons (e.g., occupied with other subjects, patient discharged) (see Figure 1). The most common reasons for refusal to participate in phase $2(n=41)$ were related to the length of the interview; the patient being too ill, weak or tired; failure to schedule or keep an appointment for the interview; or patient denial of cocaine use despite a positive urine laboratory result.

Sociodemographic and Clinical Characteristics. Table 1 presents the sociodemographic, clinical, and service use characteristics for the total sample (e.g., phase 2 participants).

The majority of the sample $(86 \% ; n=124)$ met the American Psychiatric Association's DSM-IV criteria for a lifetime diagnosis of substance abuse $(8 \% ; n=12)$ or dependence, with most $(77 \% ; n=121)$ meeting criteria for dependence (Table 1). One half of the total sample $(50 \% ; n=72)$ met criteria for a recent substance use diagnosis in the past three months. Among those with a recent substance dependence diagnosis $(n=59)$, the sample was fairly evenly divided among those with cocaine dependence $(28 \% ; n=40)$ and alcohol dependence $(10 \% ; n=15)$ while four subjects $(3 \%)$ met criteria for other drug dependence (marijuana was the other illicit drug used most often). Among the 13 patients (9\%) who met criteria for past three-month substance abuse diagnosis, seven (54\%) met criteria for alcohol abuse and six (46\%) met criteria for cocaine abuse.

As shown in Table 1, the study participants overall reported an average of 7.6 days using crack/cocaine in the past month before ED presentation (median, 4 days). More than one third of the sample reported very infrequent use of cocaine during the previous 28 days (e.g., $1-2$ days, $n=58,42 \%), 41(30 \%)$ reported cocaine use ranging from three to ten days, 30 (22\%) reported cocaine use ranging from 11 to 28 days, and nine $(7 \%)$ reported daily use of cocaine in the past 28 days (data not shown). Seven participants, despite a positive urine toxicology screen for cocaine, refused to provide data on how many days they had used cocaine in the past 28 days. Recent marijuana and alcohol use were also commonly reported by study participants (only one participant refused to answer frequency items about marijuana). Overall, the sample had moderate levels of depression from the PHQ-9

\section{TABLE 1. Sociodemographic, Service Use, Substance Use, and Psychological Characteristics of Total Sample $(n=145)$}

\begin{tabular}{|c|c|}
\hline \multicolumn{2}{|l|}{ Characteristics } \\
\hline Male (\%) & $97(67)$ \\
\hline African-American (\%) & $111(77)$ \\
\hline Married/living together (\%) & $37(26)$ \\
\hline High school educated (\%) & $89(61)$ \\
\hline Employed (\%) & $66(46)$ \\
\hline \multicolumn{2}{|l|}{ Annual income* $(\%)$} \\
\hline$<\$ 10,000$ & $32(29)$ \\
\hline$\$ 10,000-19,999$ & $42(38)$ \\
\hline$>\$ 20,000$ & $36(33)$ \\
\hline \multicolumn{2}{|l|}{ DSM-IV substance use diagnoses (\%) } \\
\hline Lifetime substance abuse & $12(8)$ \\
\hline Lifetime substance dependence & $112(77)$ \\
\hline Cocaine abuse (past 3 months) & $6(4)$ \\
\hline Cocaine dependence (past 3 months) & $40(28)$ \\
\hline Alcohol abuse (past 3 months) & $7(5)$ \\
\hline Alcohol dependence (past 3 months) & $15(10)$ \\
\hline Other drug dependence (past 3 months) & $4(3)$ \\
\hline Lifetime substance abuse treatment (formal) (\%) & $79(54)$ \\
\hline $\begin{array}{l}\text { Formal or informal past-year substance abuse } \\
\text { treatment }(\%) \dagger\end{array}$ & $30(22)$ \\
\hline Past-year mental health treatment (\%) & $28(19)$ \\
\hline Past-year depression treatment (\%) & $24(17)$ \\
\hline \multicolumn{2}{|l|}{ Needed or wanted but did not get substance } \\
\hline Antisocial personality disorder (\%) & $45(29)$ \\
\hline Mean age (SD) & $39(8.9)$ \\
\hline \multicolumn{2}{|l|}{$\begin{array}{l}\text { Mean substance use frequency (past } 4 \text { weeks) } \\
\text { (SD) }\end{array}$} \\
\hline Days using crack/cocaineł & $7.6(8.2)$ \\
\hline Days using marijuana§ & $6.5(9.2)$ \\
\hline Days drinking alcohol & $11.4(10.4)$ \\
\hline \multicolumn{2}{|l|}{ Mean depression symptom severity (SD) } \\
\hline Patient Health Questionnaire 9 total score & $10.4(7.9)$ \\
\hline \multicolumn{2}{|l|}{ Mean Brief Symptom Inventory scores (SD) } \\
\hline Depression & $7.1(6.9)$ \\
\hline Anxiety & $5.7(5.5)$ \\
\hline Global severity index & $48.5(42.7)$ \\
\hline \multicolumn{2}{|l|}{$\begin{array}{l}\text { Mean Inventory of Drug Use Consequences scales } \\
\text { (SD) }\end{array}$} \\
\hline Physical & $3.2(2.8)$ \\
\hline Interpersonal & $3.1(3.1)$ \\
\hline Intrapersonal & $4.0(3.1)$ \\
\hline Impulse & $2.3(2.3)$ \\
\hline Social responsibility & $3.9(3.0)$ \\
\hline
\end{tabular}

*Thirty five participants did not respond to this item. tEight participants did not respond to this item. \#Seven participants did not respond to this item. §One participant did not respond to this item.

total scores and relatively poor psychological functioning as indicated by the BSI scales, and their InDUC-2R scores indicated frequent social consequences resulting from substance use.

Correlates of Recent DSM-IV Substance Use Diagnosis. Comparisons among the substance use diagnostic groups defined by past three-month diagnoses of substance dependence and abuse and those with no diagnosis found no significant differences in age, gender, race, marital status, education, 
TABLE 2. Sociodemographic and Service Use Correlates of Recent DSM-IV Substance Use Diagnosis

\begin{tabular}{|c|c|c|c|}
\hline & $\begin{array}{l}\text { No DSM-IV Substance } \\
\text { Diagnosis }(n=73 ; 50 \%)\end{array}$ & $\begin{array}{l}\text { DSM-IV Substance } \\
\text { Abuse }(n=13 ; 9 \%)\end{array}$ & $\begin{array}{c}\text { DSM-IV Substance } \\
\text { Dependence }(n=59 ; 41 \%)\end{array}$ \\
\hline Mean age $( \pm S D)^{*}$ & $40.2(9.8)$ & $38(7.2)$ & $37.6(7.9)$ \\
\hline Male* & $46(63)$ & $8(62)$ & $43(73)$ \\
\hline Married/living together* & $17(23)$ & $4(31)$ & $16(27)$ \\
\hline High school educated* & $41(56)$ & $7(54)$ & $41(69)$ \\
\hline Employed* $^{*}$ & $32(43)$ & $11(85)$ & $23(39)$ \\
\hline$\$ 10,000-19,999$ & $22(42)$ & $3(30)$ & $17(36)$ \\
\hline$>\$ 20,000$ & $10(19)$ & $6(60)$ & $20(43)$ \\
\hline Lifetime substance abuse treatment (formal) $\ddagger$ & $33(45)$ & $9(69)$ & $37(63)$ \\
\hline \multicolumn{4}{|l|}{ Formal or informal past-year substance abuse } \\
\hline treatment§ & $12(17)$ & $0(0)$ & $18(32)$ \\
\hline Past-year mental health treatment* & $16(22)$ & $2(15)$ & $10(17)$ \\
\hline
\end{tabular}

All values are expressed as $n(\%)$.

${ }^{*}$ No significant difference between substance use diagnosis groups $(p>0.05)$.

$+x^{2}=7.26, d f=1, p<0.0071$.

$\ddagger \chi^{2}=4.14, d f=1, \mathrm{p}<0.0418$.

$\S \chi^{2}=3.99, d f=1, \mathrm{p}<0.0457$.

$\| \chi^{2}=17.6, d f=1, \mathrm{p}<0.0001$.

employment, and past-year mental health or depression treatment (see Table 2). Significant associations were found between substance use diagnoses and income, lifetime substance abuse treatment, formal or informal past-year substance use treatment, and selfreported need or desire for substance abuse treatment but lack of getting it in the past year. Not surprisingly, individuals with recent diagnoses were more likely to have received lifetime and recent substance abuse treatment.

Table 3 shows comparisons by recent DSM-IV substance use diagnosis for substance use and mental health characteristics. Significantly associated with the presence of a substance dependence diagnosis were greater frequency of cocaine, marijuana, and alcohol use; greater depression symptom severity assessed by the PHQ-9; and greater psychological distress according to the BSI depression, anxiety, and global scores (where higher scores indicate more problems). InDUC-2R measures of physical, interpersonal, intrapersonal, impulse, and social responsibility consequences were significantly greater for those with substance use diagnoses. Antisocial personality disorder was also significantly associated with the presence of a substance use disorder diagnosis.

\section{DISCUSSION}

Although substance use is relatively frequent among patients presenting to urban public EDs, ${ }^{1,36}$ very little is known about the spectrum of drug use or extent of psychiatric disorders and psychological problems in these nontreatment settings. We report findings from a study of ED patients admitted to a CPOU who were at low to intermediate risk for ACS and had recently used cocaine before their ED presentation for chest pain. We chose to sample a homogeneous, low to moderate ACS risk population observed and discharged from the CPOU in order to gain a better understanding of the sociodemographic and clinical characteristics of cocaine users seen and discharged entirely from an ED setting. For these patients, the ED is possibly their only contact with health care providers who have an opportunity to reinforce the dangers of continued cocaine use, and emergency physicians cannot rely on inpatient services to address and intervene regarding substance use. Indeed, with continued cocaine use, this population of patients is at high risk for progression of cardiac disease, ${ }^{22}$ and most cocaine users with chest pain have few traditional risk factors for ACS other than smoking. Thus, secondary prevention of cardiac disease in this population should focus primarily on cessation of cocaine use. Moreover, understanding the spectrum of drug use and associated problems in these patients can greatly assist the clinician in determining the need for subsequent substance abuse treatment as well as ongoing cardiac risk.

Our study found a great deal of demographic and clinical heterogeneity in this population of cocaine users. The majority of our patients were high school educated, almost one half were employed, and nearly one fourth had undergone substance abuse treatment in the past year, suggesting a potentially higher 
TABLE 3. Substance Use and Psychological Correlates of Recent DSM-IV Substance Use Diagnosis

\begin{tabular}{|c|c|c|c|c|}
\hline & $\begin{array}{c}\text { No DSM-IV } \\
\text { Substance } \\
\text { Diagnosis } \\
(n=73 ; 50 \%)\end{array}$ & $\begin{array}{c}\text { DSM-IV } \\
\text { Substance } \\
\text { Abuse } \\
(n=13 ; 9 \%)\end{array}$ & $\begin{array}{c}\text { DSM-IV } \\
\text { Substance } \\
\text { Dependence } \\
(n=59 ; 41 \%)\end{array}$ & $\begin{array}{l}\text { ANOVA Post } \\
\text { Hoc Group } \\
\text { Differences }\end{array}$ \\
\hline \multicolumn{5}{|l|}{ Substance use frequency (past 4 weeks) } \\
\hline Days using crack/cocaine* & $5.2(6.4)$ & $6.9(8.1)$ & $10.6(9.3)$ & Dependence > No SUD \\
\hline Days using marijuana*,$\dagger$ & $5.1(8.3)$ & $2.4(4.1)$ & $9.1(10.4)$ & $\begin{array}{l}\text { Dependence }>\text { No SUD } \\
\text { Dependence }>\text { Abuse }\end{array}$ \\
\hline Days drinking alcohol* & $9.6(10.1)$ & $11.0(7.5)$ & $13.7(10.9)$ & Dependence > No SUD \\
\hline \multicolumn{5}{|l|}{ Depression symptom severity } \\
\hline Patient Health Questionnaire 9 total score* & $8.3(7.5)$ & $11.4(7.6)$ & $12.6(8.1)$ & Dependence > No SUD \\
\hline \multicolumn{5}{|l|}{ Brief Symptom Inventory scores } \\
\hline Depression* & $5.3(5.2)$ & $7.3(6.7)$ & $9.4(7.6)$ & Dependence > No SUD \\
\hline Anxiety* & $4.6(5.3)$ & $6.2(4.9)$ & $6.9(5.7)$ & Dependence > No SUD \\
\hline Global severity index* & $37.5(39.1)$ & $48.1(32.8)$ & $62.1(45.5)$ & Dependence > No SUD \\
\hline \multicolumn{5}{|l|}{ Inventory of Drug Use Consequences scales } \\
\hline Physical $^{*}, \dagger$ & $2.1(2.5)$ & $2.5(1.9)$ & $4.8(2.5)$ & $\begin{array}{l}\text { Dependence }>\text { No SUD } \\
\text { Dependence }>\text { Abuse }\end{array}$ \\
\hline Interpersonal ${ }^{*}, \dagger$ & $1.8(2.7)$ & $2.9(1.8)$ & $4.7(3.1)$ & $\begin{array}{l}\text { Dependence }>\text { No SUD } \\
\text { Dependence }>\text { Abuse }\end{array}$ \\
\hline Intrapersonal ${ }^{*}, \dagger$ & $2.4(2.7)$ & $3.9(2.2)$ & $6.1(2.5)$ & $\begin{array}{l}\text { Dependence }>\text { No SUD } \\
\text { Dependence }>\text { Abuse }\end{array}$ \\
\hline Impulse*,$\dagger$ & $1.3(2.0)$ & $2.4(1.2)$ & $3.6(2.2)$ & $\begin{array}{l}\text { Dependence }>\text { No SUD } \\
\text { Dependence }>\text { Abuse }\end{array}$ \\
\hline Social responsibility* ${ }^{*} \dagger$ & $2.4(2.6)$ & $3.7(1.8)$ & $5.8(2.6)$ & $\begin{array}{l}\text { Dependence }>\text { No SUD } \\
\text { Dependence }>\text { Abuse }\end{array}$ \\
\hline Antisocial personality disorder, $n(\%)$ & $12(16)$ & $4(31)$ & $25(42)$ & $\chi^{2}=10.78, d f=1, \mathrm{p}<0.01$ \\
\hline
\end{tabular}

All values are expressed as mean (SD) unless otherwise noted.

SUD = substance use disorder diagnosis.

${ }^{*} \mathrm{p}<0.05$, substance dependence vs. no diagnosis, post hoc test for group differences

$\mathrm{tp}<0.05$, substance abuse vs. substance dependence, post hoc test for group differences.

functioning cohort than may have been previously described. Although medically these patients are grouped together into "chest pain due to cocaine use" by study design, they were not homogeneous in terms of substance use consequences, psychological distress, or depressive symptomatology or treatment history. One half of the sample met criteria for substance abuse or dependence in the past three months, primarily cocaine or alcohol dependence; on the other hand, a substantial proportion surprisingly did not meet abuse or dependence criteria and reported infrequent cocaine use. The anecdotal clinical perception of cocaine users who present to urban EDs with chest pain is that they are "addicts" or heavy users. This perception may be in part due to clinical stereotyping where clinicians are more likely to remember and classify cocaine/crack users as frequent recidivists or "frequent flyers" who may overrepresent the more severe end of the spectrum of cocaine use. ${ }^{37,38}$

Weber et al. have recommended that institutions implementing brief observation periods for patients who present to the ED with cocaine-associated chest pain also incorporate strategies for encouraging substance abuse treatment due to increased likelihood of nonfatal myocardial infarction in those who continue to use cocaine. ${ }^{22}$ Occurrence of chest pain severe enough to bring a cocaine user to the ED could provide the opportunity for brief interventions to increase problem recognition among cocaine-using individuals and increase follow-through with treatment referral. ${ }^{23,39-41}$ The concept of intervening for risky behaviors among ED patients, in particular alcohol problems, has been shown to be reasonable and effective. ${ }^{39,40}$ One step to identifying the cocaineusing patient with chest pain who may greatly benefit from substance use treatment is to discuss the extent of recent cocaine and alcohol use, both of which were strongly associated with recent substance abuse diagnoses in our sample. About one half of patients meeting abuse or dependence criteria reported they needed or wanted treatment. ED staff might consider counseling patients who report very frequent recent cocaine or alcohol use to seek assessment for possible substance abuse treatment. However, all patients with cocaine-associated chest pain should be advised to refrain from cocaine use to prevent acceleration of coronary disease.

We found that substance use consequences measured by the InDUC-2R were significantly more likely to occur among those with DSM-IV substance use disorders, and indeed the scores for patients with recent dependence are similar to those reported in treatment samples. ${ }^{33}$ This finding corroborates diagnoses derived by self-report on the SAOM and is not surprising given that the DSM-IV criteria also measure both physical and psychological consequen- 
ces of substance use. ${ }^{31}$ What we do not know is whether physical and psychological consequences of greater substance use, such as those in the DSM-IV or the InDUC-2R, are risk factors for subsequent ACS, and longitudinal data are needed to determine this issue. Interestingly, previous work by Hollander et al. ${ }^{5}$ found that demographic and clinical factors, including the frequency of cocaine use, were of little assistance to physicians in identifying/discriminating which patients with cocaine-associated chest pain are at very low risk for myocardial infarction.

About one half of our sample reported moderate to severe depressive symptomatology on the PHQ-9. ${ }^{29,30}$ BSI scores of the sample are higher than general population norms and are comparable to norms for a large outpatient psychiatry sample. ${ }^{42}$ Depressive symptomatology can be caused by intoxication and withdrawal from drugs or alcohol as well as by the psychological sequelae of substance use disorders $^{31,43-45}$; thus, any depressive symptoms observed in our sample at the time of completing the interviews may be due to a variety of reasons. Even though we did not make diagnoses of major depressive disorder because of the potential confounding effect of cocaine withdrawal, it is clear that depressive symptomatology is higher among those with substance use disorder diagnoses and may be a function of greater frequency of cocaine use. This issue warrants further investigation. In a non-cocaine use population, the PHQ-9 scores we observed for those with substance dependence diagnoses would be indicative of a major depressive disorder. Only about $20 \%$ of the sample reported seeking treatment for depression or other mental health issues in the past year.

\section{LIMITATIONS}

One limitation of this study is that the protocol, as required by the local institutional review board, did not allow for viewing medical records of patients who did not consent to participate in phase 1 of the study; thus, it was not possible to examine whether they were different from consenting patients. Furthermore, all of our data, including reports of substance use frequency, are self-report, although all but six of our sample had a positive urine screen for recent cocaine use. It is possible that patients in this study underreported the frequency of their cocaine or other drug use due to embarrassment, fear of legal repercussions, desire to minimize their problems, and/or issues related to talking with a stranger about these issues. Thus, our findings may actually reflect an underestimate of the extent of substance use severity in this sample. Nonetheless, a strength of the data presented in this study is that it represents an almost complete and consecutive enumeration of patients presenting to a CPOU with chest pain and recent cocaine use.

\section{CONCLUSIONS}

Patients with chest pain in the ED who have recently used cocaine represent a diverse spectrum of substance abuse. The occurrence of chest pain severe enough to bring a cocaine user to the ED could provide the opportunity for cocaine-using individuals to recognize the adverse and potentially life-threatening consequences of drug use and to either seek formal or informal treatment or to reduce or eliminate their cocaine use without treatment. The costs associated with ED service use and cocaine-related chest pain have been extraordinary. ${ }^{5}$ The ED serves a unique role in the health care system and many patients who use EDs, especially inner-city EDs, do not have a primary care physician, do not routinely receive medical care other than in the ED setting, are minority patients, and/or are patients of lower socioeconomic status. $^{46-49}$ Thus, patients who are reliant on such emergency care may be in greater need of prompt, seamless referral and follow-up care to interrupt the downward spiral associated with continued cocaine use and its medical and psychological complications.

The authors thank Shanti Tripathi (statistical analyst), Debbie Hodges (database manager), and Shannon Watson (administrative support).

\section{References}

1. U.S. Department of Health and Human Services. Substance Abuse and Mental Health Services Administration, Office of Applied Studies. Emergency Department Trends from the Drug Abuse Warning Network, Final Estimates 1995-2002. DAWN Series: D-24, DHHS Publication No. (SMA) 03-3780. 2003. Available at: DAWNinfo.samhsa.gov/old_dawn/pubs_94_02/ edpubs/2002final/files/EDTrendFinal02AllText.pdf. Accessed Feb 22, 2005.

2. Minor RL Jr, Scott BD, Brown DD, Winniford MD. Cocaineinduced myocardial infarction in patients with normal coronary arteries. Ann Intern Med. 1991; 115:797-806.

3. Hollander JE, Todd $\mathrm{KH}$, Green $\mathrm{G}$, et al. Chest pain associated with cocaine: an assessment of prevalence in suburban and urban emergency departments. Ann Emerg Med. 1995; 26: 671-6.

4. Weber JE, Chudnofsky CR, Boczar M, Boyer EW, Wilkerson MD, Hollander JE. Cocaine-associated chest pain: how common is myocardial infection? Acad Emerg Med. 2000; 7:873-7.

5. Hollander JE, Hoffman RS, Gennis P, et al. Prospective multicenter evaluation of cocaine-associated chest pain. Cocaine Associated Chest Pain (COCHPA) Study Group. Acad Emerg Med. 1994; 1:330-9.

6. Mittleman MA, Mintzer D, Maclure M, Tofler GH, Sherwood JB, Muller JE. Triggering of myocardial infarction by cocaine. Circulation. 1999; 99:2737-41.

7. Qureshi AI, Suri MF, Guterman LR, Hopkins LN. Cocaine use and the likelihood of nonfatal myocardial infarction and stroke. Data from the Third National Health and Nutrition Examination Survey. Circulation. 2001; 103:502-6.

8. Lange RA, Cigarroa RG, Yancy CW Jr, et al. Cocaine-induced coronary-artery vasoconstriction. N Engl J Med. 1989; 321: $1557-62$.

9. Isner JM, Estes M III, Thompson PD, et al. Acute cardiac events temporally related to cocaine abuse. N Engl J Med. 1986; 315: 1438-43. 
10. Mittleman RE, Wetli CV. Cocaine and sudden "natural" death. J Forensic Sci. 1987; 32:11-9.

11. Togna G, Tempesta E, Togna AR, Dolci N, Cebo B, Caprino L. Platelet responsiveness and biosynthesis of thromboxane and prostacyclin in response to in vitro cocaine treatment. Haemostasis. 1985; 15:100-7.

12. Rezkalla SH, Mazza JJ, Kloner RA, Tillema V, Chang SH. Effects of cocaine on human platelets in healthy subjects. Am J Cardiol. 1993; 72:243-6.

13. Zimmerman FH, Gustafson GM, Kemp HG Jr. Recurrent myocardial infarction associated with cocaine abuse in a young man with normal coronary arteries: evidence for coronary artery spasm culminating in thrombosis. J Am Coll Cardiol. 1987; 9:964-8.

14. Stenberg RG, Winniford MD, Hillis LD, Dowling GP, Buja LM. Simultaneous acute thrombosis of two major coronary arteries following intravenous cocaine use. Arch Pathol Lab Med. 1989; 113:521-4.

15. Dressler FA, Malekzadeh S, Roberts WC. Quantitative analysis of amounts of coronary arterial narrowing in cocaine addicts. Am J Cardiol. 1990; 65:303-8.

16. Moliterno DJ, Lange RA, Gerard RD, Willard JE, Lackner C, Hillis LD. Influence of intranasal cocaine on plasma constituents associated with endogenous thrombosis and thrombolysis. Am J Med. 1994; 96:492-6.

17. Tardiff K, Gross E, Wu J, Stajic M, Millman R. Analysis of cocaine-positive fatalities. J Forensic Sci. 1989; 34:53-63.

18. Hollander JE, Hoffman RS, Burstein JL, Shih RD, Thode HC Jr. Cocaine-associated myocardial infarction: mortality and complications. Cocaine-Associated Myocardial Infarction Study Group. Arch Intern Med. 1995; 155:1081-6.

19. Soderstrom CA, Dischinger PC, Smith GS, McDuff DR, Hebel JR, Gorelick DA. Psychoactive substance dependence among trauma center patients. JAMA. 1992; 267:2756-9.

20. Soderstrom CA, Smith GS, Dischinger PC, et al. Psychoactive substance use disorders among seriously injured trauma center patients. JAMA. 1997; 277:1769-74.

21. Ziedonis DM, Rayford BS, Bryant KJ, Rounsaville BJ. Psychiatric comorbidity in white and African-American cocaine addicts seeking substance abuse treatment. Hosp Community Psychiatry. 1994; 45:43-9.

22. Weber JE, Shofer FS, Larkin GL, Kalaria AS, Hollander JE. Validation of a brief observation period for patients with cocaine-associated chest pain. N Engl J Med. 2003; 348:510-7.

23. El-Guebaly N, Armstrong SJ, Hodgins DC. Substance abuse and the emergency room: Programmatic implications. J Addict Dis. 1998; 17(2):21-40.

24. Gibler WB, Runyon JP, Levy RC, et al. A rapid diagnostic and treatment center for patients with chest pain in the emergency department. Ann Emerg Med. 1995; 25:1-8.

25. Tietz Fundamentals of Clinical Chemistry, ed 4. Philadelphia, PA: WB Saunders Co., 1996.

26. Smith GR, Ross RL, Rost KM. Psychiatric outcomes module: Substance Abuse Outcomes Module (SAOM). In: Sederer LI, Dickey B (eds). Outcomes Assessment in Clinical Practice. Baltimore, MD: Williams \& Wilkins, 1996, pp 85-8.

27. Smith GR Jr, Babor T, Burnam MA, Mosley CL, Rost K, Burns B. Substance Abuse Outcomes Module: User's Manual. Little Rock, AR: University of Arkansas for Medical Sciences. Available at: www.netoutcomes.net.

28. Derogatis LR. BSI: Brief Symptom Inventory: Administration, Scoring, and Procedures Manual. Minneapolis, MN: National Computer Systems, Inc., 1993.

29. Kroenke K, Spitzer RL, Williams JBW. The PHQ-9: validity of a brief depression severity measure. J Gen Intern Med. 2001; 16:606-73.
30. Spitzer RL, Kroenke K, Williams JBW. Validation and utility of a self-report version of PRIME-MD: the PHQ primary care study. JAMA. 1999; 282:1737-44.

31. American Psychiatric Association. Diagnostic and Statistical Manual of Mental Disorders, ed 4. Washington, DC: American Psychiatric Association, 1994.

32. Miller WR, Tonigan JS, Longabaugh R. The Drinker Inventory of Consequences (DRINC): An Instrument for Assessing Adverse Consequences of Alcohol Abuse, Test Manual. Volume 4, Project MATCH Monograph Series. Rockville, MD: National Institute on Alcohol Abuse and Alcoholism, 1995.

33. Smith GR, Burnam MA, Mosley CL, Hollenberg JA, Mancino M, Grimes W. Measuring Substance Abuse Treatment and Outcomes: The Reliability and Validity of the Substance Abuse Outcomes Module. In press.

34. Cottler LB, Robins LN, Helzer JE. The reliability of the CIDISAM: a comprehensive substance abuse interview. Br J Addict. 1989; 84:801-14.

35. McLellan AT, Kushner H, Metzger D, et al. The fifth edition of the Addiction Severity Index. J Subst Abuse Treat. 1992; 9: 199-213.

36. Curran GM, Sullivan G, Williams DK, et al. Emergency department use of persons with comorbid psychiatric and substance abuse disorders. Ann Emerg Med. 2003; 41:659-67.

37. Ritson EB. Alcohol, drugs, and stigma. Int J Clin Pract. 1999; 53:549-51.

38. Miller NS, Sheppard LM, Colenda CC, Magen J. Why physicians are unprepared to treat patients who have alcoholand drug-related disorders. Acad Med. 2001; 76:410-8.

39. Bernstein E, Bernstein J, Levenson S. Project ASSERT: an EDbased intervention to increase access to primary care, preventive services, and the substance abuse treatment system. Ann Emerg Med. 1997; 30:181-9.

40. Longabaugh R, Woolard RE, Nirenberg TD, et al. Evaluating the effects of a brief motivational intervention for injured drinkers in the emergency department. J Stud Alcohol. 2001; 62:806-16.

41. Dunn C, Deroo L, Rivara FP. The use of brief interventions adapted from motivational interviewing across behavioral domains: a systemic review. Addiction. 2001; 96:1725-42.

42. Derogatis LR. Brief Symptom Inventory (BSI) Administration, Scoring and Procedures Manual, ed 4. Minneapolis, MN: NCS Pearson, Inc., 1993.

43. Brown RA, Monti PM, Myers MG, et al. Depression among cocaine abusers in treatment: relation to cocaine and alcohol use and treatment outcome. Am J Psychiatry. 1998; 155: 220-5.

44. Brown SA, Schuckit MA. Changes in depression among abstinent alcoholics. J Stud Alcohol. 1988; 49:412-7.

45. Nunes EV, Deliyannides D, Donovan S, McGrath PJ. The management of treatment resistance in depressed patients with substance use disorders. Psychiatr Clin North Am. 1996; 19:311-27.

46. Baker DW, Stevens CD, Brook RH. Patients who leave a public hospital emergency department without being seen by a physician: causes and consequences. JAMA. 1991; 266: 1085-90.

47. Bindman AB, Grumbach K, Keane D, Rauch L, Luce JM. Consequences of queuing for care at a public hospital emergency department. JAMA. 1991; 266:1091-6.

48. Grumbach K, Keane D, Bindman A. Primary care and public emergency department overcrowding. Am J Public Health. 1993; 83:372-8.

49. Pane GA, Farner MC, Salness KA. Health care access problems of medically indigent emergency department walk-in patients. Ann Emerg Med. 1991; 20:730-3. 